\title{
FABRICATION OF ATOM TRAPPING CHIPS WITH FEEDTHROUGH INTERCONNECTS FOR APPLICATIONS IN ATOM OPTICS
}

\author{
H.C.(Rick) Chuang $^{1}$, D.Z. Anderson ${ }^{2}$, and V.M. Bright ${ }^{1}$ \\ ${ }^{1}$ Department of Mechanical Engineering, University of Colorado, Boulder, Colorado, USA \\ ${ }^{2}$ Department of Physics, University of Colorado, Boulder, Colorado, USA
}

\begin{abstract}
This paper presents a new method to fabricate the feedthrough vias in atom trapping chips used in ultra-high-vacuum (UHV) atom-optics cells for Bose-Einstein Condensation (BEC) experiments. A process was developed which uses silicon nitride thin film and copper electroplating to seal the vias. The atom trapping chips offer simple microfabrication and reduced volume of the atom trapping cell. The results demonstrate that 8 Amps current can be conducted through the vias while the vacuum can be held under $4 \times 10^{-11}$ Torr at room temperature. The via fabrication yield after anodic bonding $\left(425^{\circ} \mathrm{C}\right)$ is $97 \%$, a considerable change over our prior results of $30 \%$ using a different process [1].
\end{abstract}

\section{INTRODUCTION}

BECs have been realizable for several years now and some of the advantages of microscopic magnetic traps for cold atoms on a chip have been described [2, 3]. W. Hansel [2] demonstrated that the formation of BEC can be simplified using a magnetic atom trap on a chip in a glass cell under UHV. The electrical connections to the chip were made by wires soldered on the connector inside the cell. The wires and solder areas were found to be vulnerable, resulting in an unreliable vacuum seal. Previously our group [4] proposed a different portable atom trapping chip system in which the chip itself forms one wall of the vacuum cell by attaching a mirrored silicon plate (50 $\mu \mathrm{m}$ thick) anodically bonded to the cell (Figure 1). However, the electrical connection is realized by pin connectors along the chip edges which again require much soldering in a small area.

In the last decade, a large number of sensors and actuators have been developed using MEMS processes. Each application puts specific requirements on the package dimensions, materials, cost and reliability. Through-wafer electrical connections are now becoming increasingly important for three-dimensional integrated circuits, microelectromechanical systems packaging and radio-frequency components. Therefore, wafer through-hole interconnect technology has been studied extensively [5,6]. Most research teams report their results on the formation of through-wafer metal plugs using copper electroplating techniques. However, all of the previous works reviewed simply require electrical conduction from one side of substrate to the other side; none of them require an ultra-high vacuum (UHV) seal (below $10^{-9}$ Torr) on the feedthrough vias. Here, we propose the new fabrication of feedthrough atom trapping chips based on a simple microfabrication process.

In this paper, we present our feedthrough atom trapping chip fabrication process by copper electroplating inside through-wafer vias. With the new feedthrough atom chips, the atom chips can be anodically bonded to the glass cell instead of using the epoxy. Therefore, the glass cell baking temperature can be raised up to $400^{\circ} \mathrm{C}$ without any leaking problems, giving a much better vacuum quality for BEC experiments. After the glass cell baking process, a custom connector can be directly clipped on the back side of the atom chip for electrical connections, thus eliminating any soldering.

\section{FABRICATION PROCESS}

The atom chip, Figure 2, is made from a double side polished, 3 inch diameter silicon wafer (100 cut, $380 \mu \mathrm{m}$ thick). A $1 \mu \mathrm{m}$ thick low stress silicon nitride layer (LPCVD) was grown on both sides of the wafer as an insulating layer. Etch windows on the silicon nitride layer are patterned by RIE and the electrical feedthrough vias (120 $\mu \mathrm{m} \times 120 \mu \mathrm{m}$ square) are then etched through the wafer by $\mathrm{KOH}$ ( $30 \%$ in DI water, $80^{\circ} \mathrm{C}$ ). After $\mathrm{KOH}$ etching, the $1 \mu \mathrm{m}$ thick silicon nitride thin film was left on the other side of the substrate. An adhesion layer, chromium (15 nm), and a gold layer $(400 \mathrm{~nm})$ were evaporated on both sides of the substrate and inside the vias as a mask layer for HF etching. The front side anodic bonding areas were patterned and the silicon nitride layer is etched away by HF (48\%) in these locations. The rest of silicon nitride layer was protected by the gold mask. After HF etching, the gold layer and chromium layer were stripped by aqua regia solution and CR-7. A $100 \mathrm{~nm}$ oxide layer was thermally grown on both sides of the substrate and inside the vias as an insulting layer between vias. An adhesion layer, titanium (30 nm), and a copper seed layer (250 nm) were evaporated on both sides of the substrate and inside the vias. A photoresist (AZ 4620) was spin coated on both sides of the substrate. The backside pads and via openings were patterned. A $15 \mu \mathrm{m}$ thick copper layer was then plated by electroplating. The copper electroplating current density was set to $40 \mathrm{~mA} / \mathrm{cm}^{2}$ and the deposition rate was around $0.5 \mu \mathrm{m} / \mathrm{min}$. The total plating time was 30 mins. After backside pad plating, the front side via openings were patterned and then the copper seed layer and titanium adhesion layer were etched away using copper and titanium etchants. The copper etchant consisted of $500 \mathrm{ml}$ DI water, $5 \mathrm{ml}$ Sulfuric acid and $60 \mathrm{~g}$ Microetch 85 powder from Technic, Inc. The etching rate was approximately $0.25 \mu \mathrm{m} / \mathrm{min}$ at room temperature. The titanium etchant consisted of 40 parts DI water, 1 part Nitric acid and 1 part Hydrofluoric acid. The silicon nitride layer $(1 \mu \mathrm{m})$ and oxide layer $(100 \mathrm{~nm})$ inside the front side via opening were removed by RIE. The atom chip wires (100 $\mu$ m wide) were patterned on the front side and $15 \mu \mathrm{m}$ thick copper was deposited by copper electroplating with the same plating parameters setup mentioned previously. After the vias were sealed by copper electroplating, the adhesion layer, titanium and copper seed layers were stripped off the chip. The final step was to pattern and etch away the oxide layer for anodic bonding of the chip to the glass cell of the portable vacuum system. The main steps of the fabrication process are described in Figure 2 and the microscopic cross-sectional images (optical microscope) of the diced fabricated vias are shown in Figure 3. Microscope images of the front sides of fabricated vias are shown in Figure 4 and photographs of atom trapping chip anodically bonded to a glass cell are shown in Figure 5.

\section{VACUUM GLASS CELL CONSTRUCTION}

After anodic bonding, a miniature BEC glass system assembled with atom trapping chip was attached to the pumping station as shown in Figure 6. The glass cell is assembled without epoxy being used in the vacuum. The anodic bonding process makes this possible, and allows the vacuum cells to be processed at much higher temperatures than was possible with earlier cell fabrication techniques [4]. The atom trapping chip with electrical feedthrough vias simplifies electrical connections to the vacuum regions of the system. 

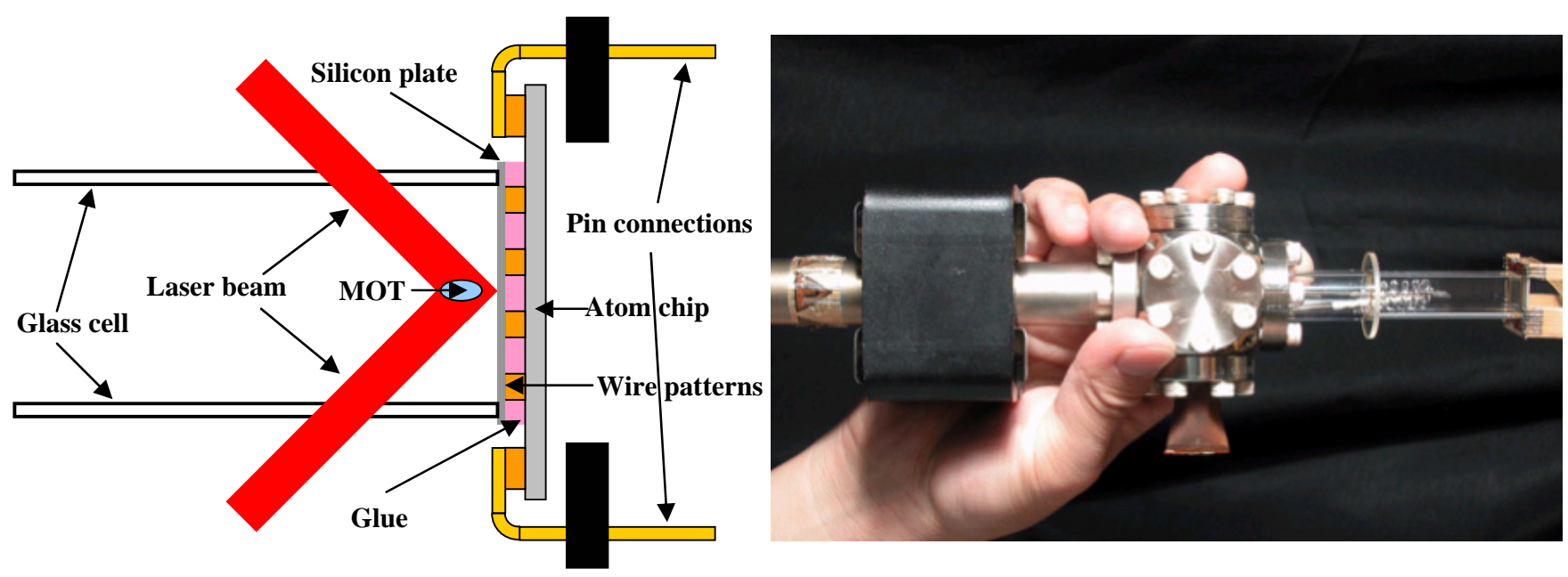

Figure 1: Schematic drawing and photograph of the portable BEC vacuum system in which MOT is created (Magneto-Optical Trap).

Front side wire patterning

Silicon wafer (100 cut, 380 um thick)

Low Stress LPCVD Silicon Nitride (1um)

Silicon Nitride patterned by RIE

$\mathrm{KOH}$ etching $\left(30 \%\right.$ in DI water, $\left.80^{\circ} \mathrm{C}\right)$

$\mathrm{Au} / \mathrm{Cr}(400 \mathrm{~nm} / 15 \mathrm{~nm})$ evaporation

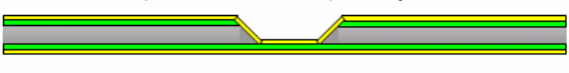

Front side anodic bonding area patterning

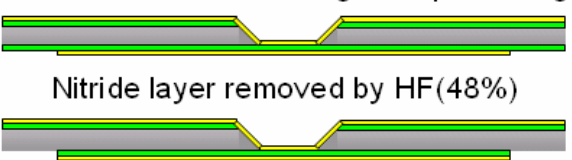

$\mathrm{Au}$ and $\mathrm{Cr}$ layer stripped

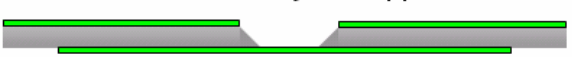

Thermal Oxidation $(100 \mathrm{~nm})$ at $\left.1100^{\circ} \mathrm{C}\right)$

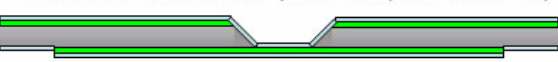
$\mathrm{Cu} / \mathrm{Ti}(250 \mathrm{~nm} / 30 \mathrm{~nm})$ seed layer evaporation

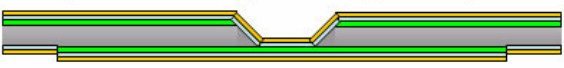

Back side pad patterning and via opening

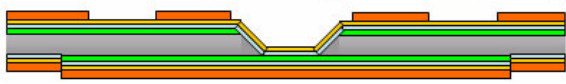

Copper electroplating (15 um)

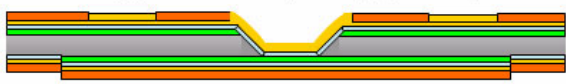

Remove front side $\mathrm{Cu} / \mathrm{Ti}$ seed layer

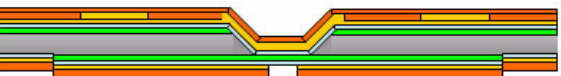

Nitride layer and $\mathrm{SiO}_{2}$ removed by RIE

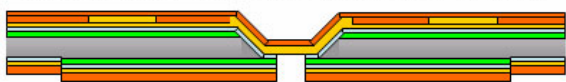

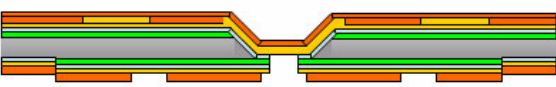

Copper electroplating (15 um)

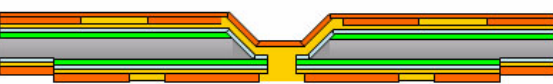

PR stripped and Cu/Ti seed layer etched away

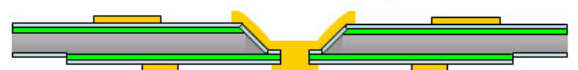

Front side anodic bonding area patterning

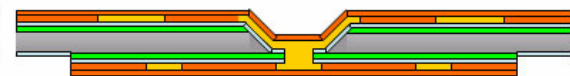

$100 \mathrm{~nm}$ oxide layer removed by HF (48\%)

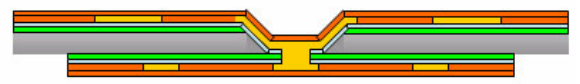

PR stripped by acetone

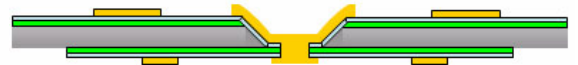

Figure 2: Fabrication process for creating feedthrough atom trapping chips.

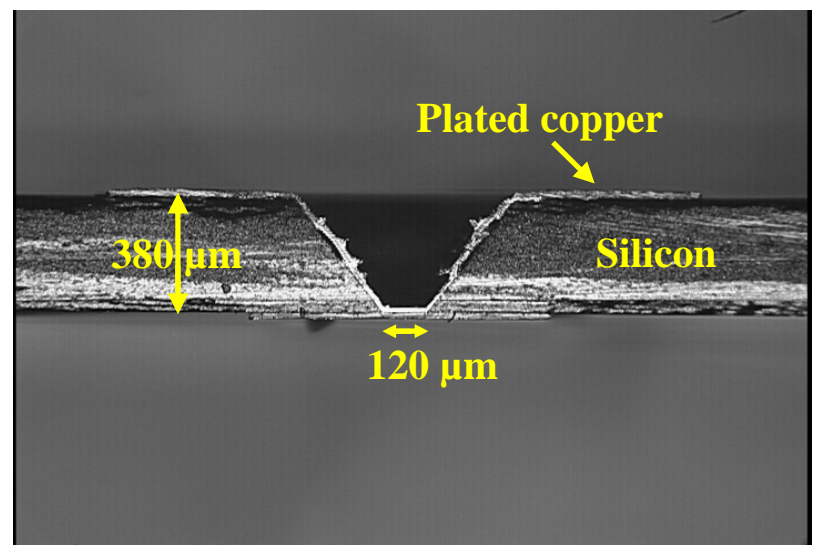

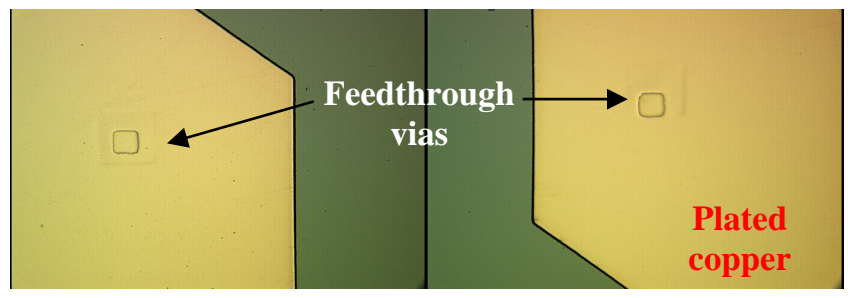

Figure 4: Microscope images of the fabricated vias on the front side of the atom chip.

Figure 3: Cross-sectional view of the diced vias. 

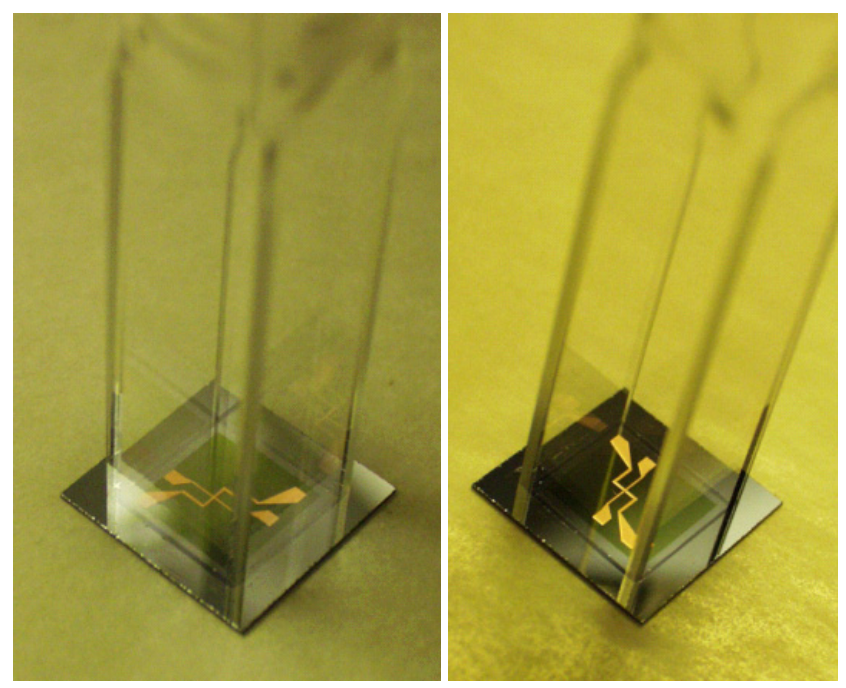

Figure 5: Photographs of atom trapping chip anodically bonded to a glass cell.

As show in Figure 6 the vacuum chamber has 4 general parts: the atom chip, the BEC cell, the center six way flange, and the 2D MOT cell. The 2D MOT cell is made from a $1 \mathrm{~cm}$ Pyrex flourimeter cell (Starna Cells 3-Px-10). The cell is lapped and polished on the open end, and a rubidium source tube is glass blown to one of the side walls of the cell. The source tube is a $12 \mathrm{~mm}$ Pyrex tube that is closed by a Pyrex disk with four pin press electrical feedthroughs. Attached to the pin presses are a non-evaporable getter (SAES getters HI/7-6) and a SAES rubidium dispenser. The getter is used to pump the hydrogen released by the $\mathrm{Rb}$ dispenser and to help maintain the vacuum in the 2D MOT cell. The 2D MOT cell is joined to the BEC cell and vacuum pumps by the center vacuum flange. The flange itself is a 6 way mini-conflat cube (Kimball Physics MCF133-SC6). A 2l/s ion pump mounted perpendicular to the MOT cells maintains the pressure in the BEC cell. Additionally a second non-evaporable getter (SAES getters HI 7/6) is connected to a conflat mounted power feedthough and is used in lieu of a titanium sublimation pump. A copper pinch off tube is brazed to a third conflat flange. The pinch off tube is attached to a vacuum pumping station for the initial pump down and bakeout. After bakeout the copper tube is pinched off so the vacuum cell can be separated from the pumping station. The BEC cell is made by glass blowing a custom made (New Era Enterprises) square 1cm ID Pyrex cell to a Pyrex glass to metal mini-conflat adaptor. The other end of the cell is lapped and polished for anodic bonding. A fabricated silicon atom chip with UHV electrical vias is anodically bonded to this polished surface.

\section{ELECTRICAL AND VACUUM TEST RESULTS}

After the feedthrough atom trapping chips were made, a quick vacuum test was performed by a helium vacuum detector in order to check if there were any immediate leaks on the feedthrough vias. The atom chip was placed on a chuck with an O-ring and the leak detector is pumped to high vacuum. Once the vacuum reaches a reasonable value $\left(1 \times 10^{-6} \sim 1 \times 10^{-7}\right.$ Torr), the helium gas probe is placed close to the vias in order to check for leaking. If the vias leak, the helium detector detects helium that comes through the feedthrough vias and the vacuum pressure readout of the helium detector will increase immediately. No leaking of the feedthrough vias was detected.

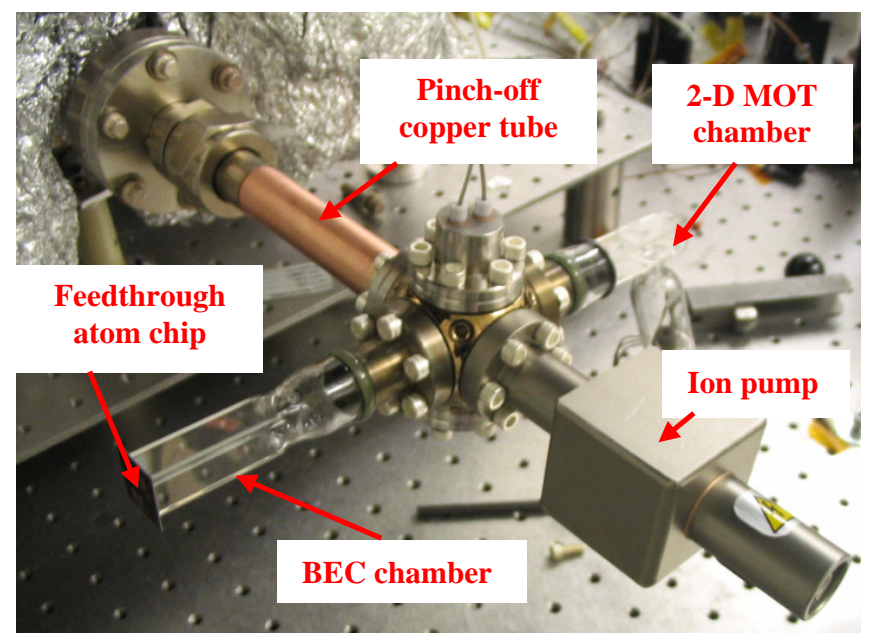

Figure 6: A miniature BEC system attached to the pumping station.

\section{Resistance measurement}

The BEC experiments require high current (5 Amps) to run through the feedthrough vias. Therefore, resistance measurements become important to examine the ability of fabricated feedthrough vias to handle such high currents. In order to deal with the problem of contact resistance when measuring the resistance from the vias, a four point resistance measurement method was performed. The resistances across the feedthrough vias were measured by a Multimeter (Keithley, 2002 Multimeter). The measurement results are shown in Figure 7. All of the measured resistances from randomly selected 20 vias are all below 0.1 ohms, which is low enough to allow sufficient current for the BEC experiments.

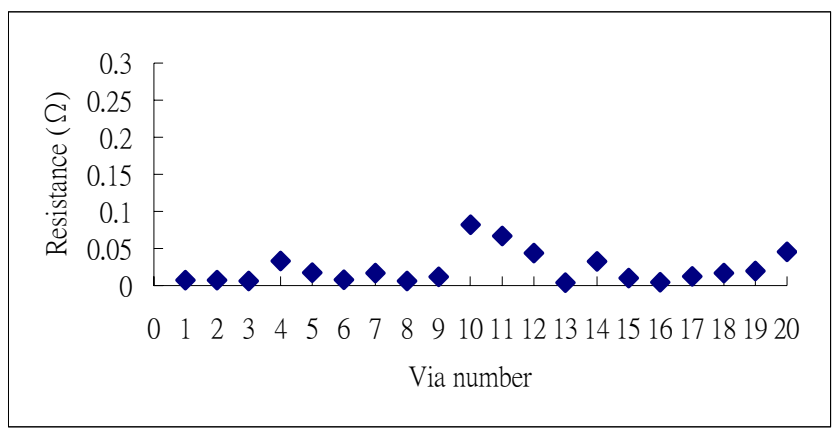

Figure 7: Resistances measurement from 20 vias (The measured resistances of the 20 vias are all below $0.1 \Omega$ ).

\section{Current measurement}

In order to create adequate magnetic fields to trap atoms in BEC experiments, no more than 5 Amps current running through the copper wires is required, and thus through the feedthrough vias as well. Five randomly selected vias on the atom chip were soldered to wires on the bonding pads of the atom chip. Current, ranging from zero to 8.7 Amps, was applied through soldered wires by a high current power supply (Sorensen, DCS8-125E) for 1 minute. The test results show that all of the five feedthrough vias survived during the high current test. From this current test results, 8 Amps of current can be successfully run through the feedthrough vias for 1 minute without burning them out. Therefore, all of the tested vias were suitable for BEC experiments, which run typically under 1 minute. The measured voltage as a function of applied current is shown in Figure 8. 


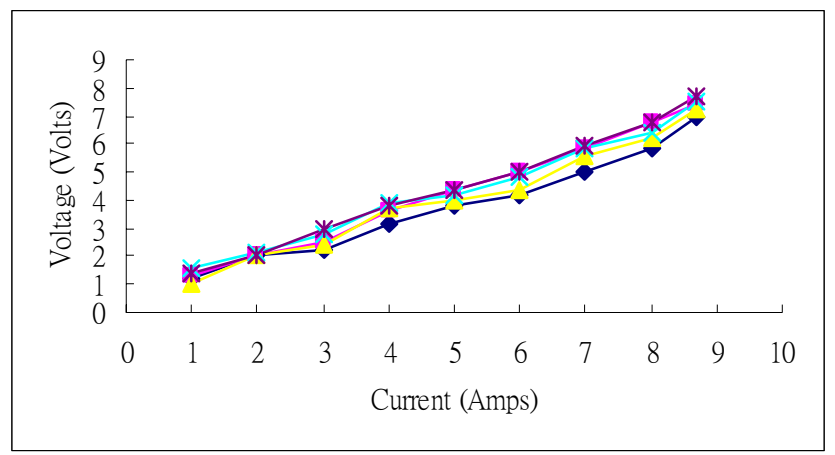

Figure 8: The measured voltage as a function of applied current for 5 feedthrough vias.

In addition to current tests, the measurement of the vias life time with different currents applied was also performed. A current range from 10 to 13 Amps was applied to four randomly selected feedthrough vias until they burned out. The measurement results are shown in Figure 9. In Figure 9, vias with 10 and 11 Amps of current applied lasted longer than 1 minute which satisfies the requirement of BEC experiments, 1 minute. However, running such high current for BEC experiments is risky since the life time is very close to 1 minute. The other vias with 12 and 13 Amps of current applied burned out after few seconds of testing.

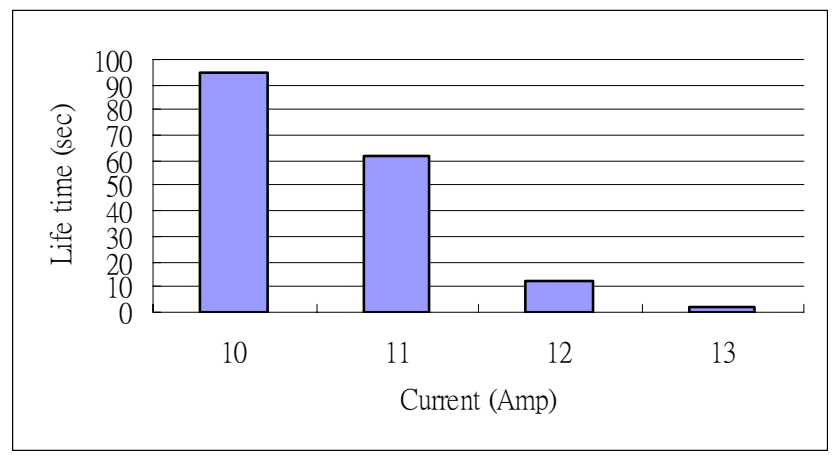

Figure 9: Feedthrough vias life time measurement with different applied current.

\section{Pressure Measurement}

After the miniature BEC system was assembled with all the components, including the atom chip, it was attached to the vacuum pumping station for initial pump down and bakeout. The whole BEC system was baked out at $350{ }^{\circ} \mathrm{C}$ for 80 hours in order to remove contamination inside the glass cell. The overall pumping time was around 3 days to reach cell pressure of $4 \times 10^{-11}$ Torr, which is low enough to pinch off for BEC experiments. No leak was found from the feedthrough vias and anodic bonding areas during the pump down and bakeout process. Next, the miniature BEC system was pinched off after the cell pressure reached $4 \times 10^{-11}$ Torr. The measured pressure curve as a function of time is shown in Figure 10. In Figure 10, there were sharp pressure peaks every 4 hours. This is because of the action of a titanium sublimation pump, which was triggered every 4 hours. When the pump was triggered, titanium was released into the cell to absorb hydrogen and then was pumped away by a turbo pump (Varian, Turbo-V70LP). After pumping for 76 hours, the bakeout process was complete and the BEC cell began to cool down, thus the cell pressure dropped substantially as shown in Figure 10. Meanwhile, the ion pump on the BEC system was activated to maintain the pressure inside the cell. At 60 hours, the BEC system was switched from the turbo pump to an Ion pump so that the system can be separated from the pumping station after pinch off. At switchover, a jump can be seen in the pressure curve. After couple hours, however, the ion pump was able to return the cell pressure to $4 \times 10^{-11}$ Torr at room temperature and it is low enough to pinch off for BEC experiments. The atom chip vias were able to hold the pressure during the entire pump and bake out process.

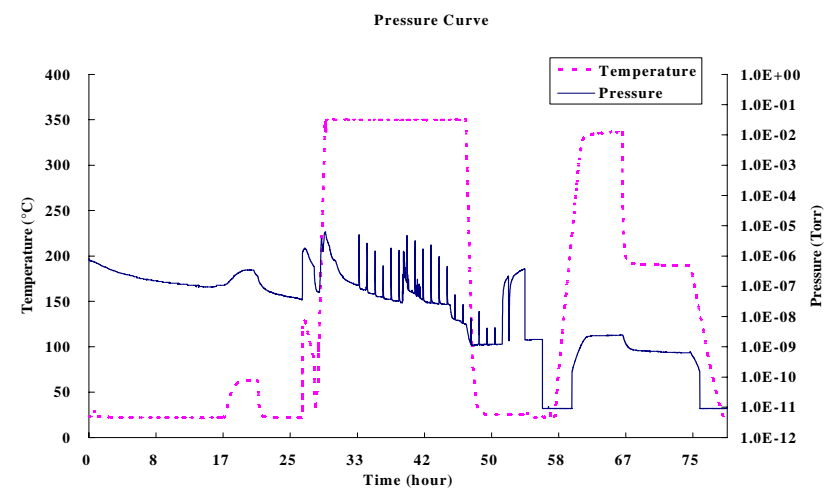

Figure 10: Pressure curve as a function of BEC cell baking time.

\section{CONCLUSIONS}

A fabrication process for the purpose of creating feedthrough atom trapping chips for BEC experiments has been developed. The fabricated feedthrough vias and anodically bonded areas were successfully tested by a helium vacuum detector. A feedthrough via fabrication yield rate of $97 \%$ was achieved after anodic bonding (required heating to $425{ }^{\circ} \mathrm{C}$ ). The resistances of 20 random feedthrough vias were measured by a four point resistance measurement method and were all below 0.1 ohms. Electrical current measurement showed that at least 8 Amps can be conducted through the vias with no leaking under vacuum. After baking out the cell $\left(350^{\circ} \mathrm{C}\right)$ on the vacuum pumping station, the final cell pressure of $4 \times 10^{-11}$ Torr at room temperature was achieved. The cell was then pinched off to maintain the vacuum for BEC experiments.

\section{REFERENCES}

[1] H.C. Chuang, T.K. Hakala, D.Z. Anderson, and V.M. Bright, "Fabrication of feedthrough atom trapping chips", Technical Digest of the $14^{\text {th }}$ International Conference on Solid-State Sensors, Actuators and Microsystems (TRANSDUCERS 2007) Lyon, France, June 10-14, Vol. 2, pp. 2059-2062.

[2] W. Hansel, P. Hommelhoff, T.W. Hansch, and J. Reichel, "Bose-Einstein condensation on a microelectronic chip", Nature, vol. 413, pp. 498-501 (2001).

[3] D. Muller, D.Z. Anderson, R.J. Grow, P.D.D. Schwindt, and E.A. Cornell, "Guiding neutral atoms around curves with lithographically patterned current-carrying wires”, Phys. Rev. Lett., vol. 83, pp. 5194 (1999).

[4] S.W. Du, M.B. Squires, V.M. Bright, K. Reichel, T.W. Hansch, and D.Z. Anderson, "Atom-chip Bose-Einstein condensation in a portable vacuum cell”, Phys. Rev. A, vol. 70, 053606 (2004).

[5] N.T. Nguyen, E. Boellaard, N.P. Pham, V.G. Kutchoukov, G. Craciun and P.M. Sarro, "Through-wafer copper electroplating for three-dimensional interconnects”, J. Micromech. Microeng., vol. 12, pp.395-399 (2002).

[6] V.G. Kutchoukov, M. Shikida, J.R. Mollinger and A. Bossche, "Through-wafer interconnect technology for silicon”, J. Micromech. Microeng., vol. 14, pp.1029-1036 (2004). 